\title{
EDITORIAL
}

For reprint orders, please contact: reprints@futuremedicine.com

\section{A promising light for an impossible disease: miRNAs in malignant gliomas}

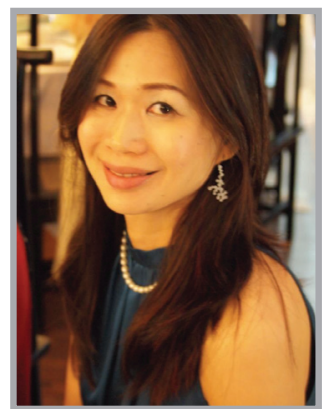

Yin Yee Sharon

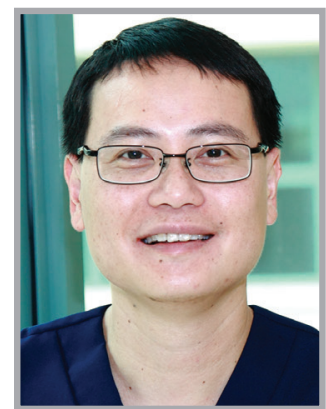

Wai Hoe $\mathrm{Ng}^{* 1,2}$

Low'

Malignant glioma remains an elusive tumor to manage despite advances in the understanding of its biology. The past few decades has shed much light into the disease's mechanisms since the days of Bailey and Cushing's insightful classification of these tumors - glioblastoma multiforme (GBM) - in 1926 [1], and attempts at radical hemispheric resection for affected patients by Dandy in 1928 [2]. In 2005, the landmark paper by Stupp et al. altered the course of history of malignant gliomas with the establishment of the 'Stupp regimen' as standard of care for newly diagnosed patients [3]. Although the combination of surgical resection, followed by concurrent radiation therapy and temozolomide (TMZ) has conferred better survival, the overall prognosis for these patients remains poor. Tumor recurrence is an inevitable reality. At present, median survival is between 12 and 18 months [3].

Recently, there has been growing evidence for the role of miRNAs in various tumors, including gliomas. miRNAs are small, highly-conserved, nonproteincoding RNAs that function as gene expression regulators at the translational level [4].
The discovery of miRNAs has provided us with a deeper insight into gene expression regulation. They have been found to play important roles in normal mammalian cell development, proliferation and death $[5,6]$. Conversely, anomalous miRNA expression has been found to be associated with cancer initiation, progression and resistance to therapeutics. Even more significantly, evidence so far has demonstrated that miRNA profiles appear to be cell type- and disease-specific key factors for disease identification, prognostication and response to treatment monitoring [6].

In the context of malignant gliomas, profiling work has demonstrated consistency in miRNAs whose expression differs from surrounding brain tissue [7]. Furthermore, Lages et al. show that there are specific miRNAs responsible for different malignant glioma subtypes [8] - a leap forward from the current WHO histopathological classification [9]. Work achieved in the field of glioma molecular biology has established that GBM tumors are known to possess aberrant cell signaling and networking pathways due to mutated growth receptors, silenced tumor suppressor genes or

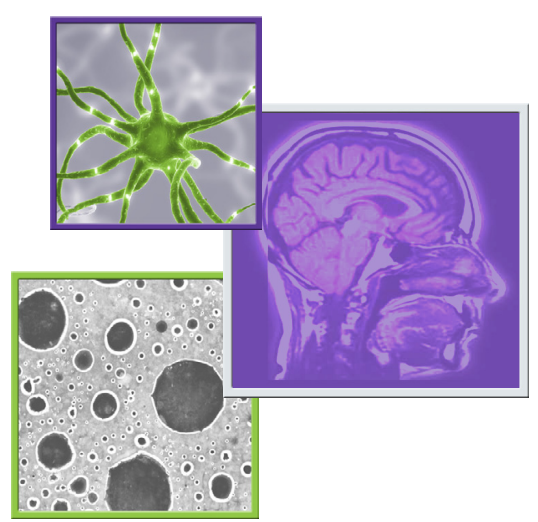

“...anomalous miRNA expression has been found to be

associated with cancer initiation, progression and resistance to therapeutics."

'Department of Neurosurgery, National Neuroscience Institute, 11 Jalan Tan Tock Seng, Singapore 308433, Singapore

${ }^{2}$ Duke-NUS Graduate Medical School, Singapore

*Author for correspondence: Tel.: +65 6357 7191; Fax: +65 6357 7120; wai_hoe_ng@nni.com.sg

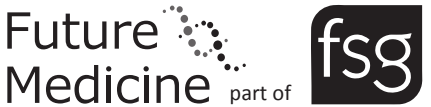




\section{“...miRNA approaches show great promise to improve current clinical management in this difficult disease. By improving our understanding of this heterogeneous tumor at a genomic level, miRNAs may hold the answers toward targeted therapy in this era of personalized medicine"}

upregulated oncogenes. All these add layers of complexity for the use of effective chemotherapeutics. However, certain miRNAs have been identified in such entities, for example, miR-7 in EGF receptor expression [10], miR-21 in matrix metalloproteinase inhibition [11] and miR-296 in proangiogenic VEGF receptor-2 expression [12]. Hence, miRNAs can provide further insight into the disease, beyond current understanding.

At present, there is no screening tool for GBM patients treated with adjuvant chemoradiotherapy. Most centers still rely on follow-up interval MRI with gadonilium contrast in order to monitor response to treatment. This is a costly modality with some limitations, such as the possibility of pseudoprogression [13], which is seen on the scans and may cast doubt on the diagnosis. miRNAs have been effectively detected in blood and tissue samples for other diseases; hence, similar techniques may be applied to GBM patients. There are advantages of using miRNAs as biomarkers in this context. Firstly, owing to their small size and stem-loop structure, miRNAs are more stable and less susceptible to degradation during fixation and sample processing. Next, the probability of discovering reliable biomarkers from approximately 1000 miRNA candidates will be higher than from over 40,000 genes. In their study, Yu et al. report plasma levels of miR-21, miR-128 and miR-342-3p being altered in GBM patients compared with normal, healthy controls [14]. In addition, the observations of Slaby et al. illustrate that there is a trend of downregulation of miRNA-181b and miRNA-181c in patients with better response to concomitant radiation therapy and TMZ, in comparison to those with disease progression [15]. Overall, there is much potential for the future use of miRNAs to augment current patient monitoring.

A key hallmark of GBM is its notoriety for recurrence, owing to an innate resistance to chemo- and radio-therapy. It is an accepted fact that acquired TMZ resistance occurs in more than $90 \%$ of recurrent malignant gliomas [16]. The topic of TMZ resistance is synonymous with the DNA repair enzyme, $O^{6}$-methylguaninemethyltransferase (MGMT). To date, MGMT is the best characterized modulator of chemoresistance in GBM. Interestingly, the bioinformatic analysis by Zhang et al. of potential genes regulated by miR-181d, suggested MGMT to be a downstream target. They showed that transfection of miR-181d in a glioma cell model effected downregulation of MGMT mRNA and protein expression [17]. Furthermore, there have been published reports of certain miRNAs implicated in TMZ resistance by various groups. For example, in vitro work by Ujifuku et al. found miR-195, miR-455 and miR-10a* to be upregulated in TMZ-resistant glioma cells [18]. Radioresistance adds another barrier to treatment efficiency in GBM. Not surprisingly, Niemoeller et al.'s analysis of human glioma cells demonstrated upregulation of miRNA clusters after treatment with clinically relevant radiation doses [19]. These results are somewhat expected as miRNAs can also be targets of molecular therapy, and those involved in apoptosis or regulating the expression of specific genes may induce the apoptosis of tumor cells exhibiting radioresistance [20].

Malignant gliomas are composed of a heterogeneous population of differentiated and nondifferentiated cells. Compared with other primary tumors, they display less sensitivity to adjuvant treatment, resulting in disease recurrence. We are now aware of a subpopulation of glioma stem cells (GSCs) with higher tumorigenic potential that contributes significantly to this inevitability [21]. These GSCs exist in a tumor microenvironment where they are often unaffected by standard therapeutics. Research in the field of GSCs have found certain miRNAs to be associated with their maintenance and propagation [16]. Therefore, by using these miRNAs as modulators, strategies targeting GSCs within their protected niche may be clinically relevant. For years, we have known the blood-brain barrier as an infallible wall, preventing many chemotherapeutics from effectively reaching the brain tumor of interest. The benefits of miRNAs' small size and stability are now being used as developmental tools for novel miRNA-based treatments [22].

\section{Conclusion \& future perspective}

Taken together, especially considering the complexities of GBM, miRNA approaches show great promise to improve current clinical management in this difficult disease. By improving our understanding of this heterogeneous tumor at a genomic level, miRNAs may hold the answers toward targeted therapy in this era of personalized medicine. In 2006, Rutka et al. wrote an inspiring review entitled 'Gliomas: quo vadis' [23]. Today, nearly a decade later, with the versatile input of miRNAs, we can perhaps, optimistically, look forward to 'Gliomas: ad locum meliorem? 


\section{Financial \& competing interests disclosure}

The authors have no relevant affiliations or financial involvement with any organization or entity with a financial interest in or financial conflict with the subject matter or materials discussed in the manuscript. This includes employment, consultancies, honoraria, stock ownership or options, expert testimony, grants or patents received or pending, or royalties.

No writing assistance was utilized in the production of this manuscript.

\section{References}

1 Bailey P, Cushing H. A Classification of the Tumors of the Glioma Group on a Histogenic Basis with a Correlated Study of Prognosis. JB Lippincott Co., Philadelphia, PA, USA (1926).

2 Dandy WE. Removal of right cerebral hemisphere for certain tumors with hemiplegia. JAMA 90, 823-825 (1928).

3 Stupp R, Mason WP, van den Bent MJ et al. Radiotherapy plus concomitant and adjuvant temozolomide for glioblastoma. N. Engl. J. Med. 352(10), 987-996 (2005).

4 Vasudevan S, Tong Y, Steitz JA. Switching from repression to activation: microRNAs can up-regulate translation. Science 318(5858), 1931-1934 (2007).

5 Lynam-Lennon N, Maher SG, Reynolds JV. The roles of microRNA in cancer and apoptosis. Biol. Rev. Camb. Philos. Soc. 84(1), 55-71 (2009).

6 Bartels CL, Tsongalis GJ. MicroRNAs: novel biomarkers for human cancer. Clin. Chem. 55(4), 623-631 (2009).

7 Silber J, Lim DA, Petritsch C et al. miR-124 and miR-137 inhibit proliferation of glioblastoma multiforme cells and induce differentiation of brain tumor stem cells. BMC Med. 6, 14 (2008).

8 Lages E, Guttin A, El Atifi M et al. MicroRNA and target protein patterns reveal physiopathological features of glioma subtypes. PloS One 6(5), e20600 (2011).

9 Louis DN, Ohgaki H, Otmar DW, Webster KCE. WHO Classification of Tumours of the
Central Nervous System. IARC, Lyon, France (2007).

10 Kefas B, Godlewski J, Comeau L et al. MicroRNA-7 inhibits the epidermal growth factor receptor and the Akt pathway and is down-regulated in glioblastoma. Cancer Res. 68(10), 3566-3572 (2008).

11 Gabriely G, Wurdinger T, Kesari S et al. MicroRNA 21 promotes glioma invasion by targeting matrix metalloproteinase regulators. Mol. Cell. Biol. 28(17), 5369-5380 (2008).

12 Wurdinger T, Tannous B, Saydam O et al. miR-296 regulates growth factor receptor overexpression in angiogenic endothelial cells. Cancer Cell 14(5), 382-393 (2008).

13 Brandsma D, Stalpers L, Taal W, Sminia P, van den Bent MJ. Clinical features, mechanisms, and management of pseudoprogression in malignant gliomas. Lancet Oncol. 9 (5), 453-461 (2008).

14 Yu J, Cai X, He J, Zhao W, Wang Q, Liu B. Microarray-based analysis of gene regulation by transcription factors and microRNAs in glioma. Neurol. Sci. doi:10.1007/s10072012-1228-1 (2012) (Epub ahead of print).

15 Slaby O, Lakomy R, Fadrus $P$ et al. MicroRNA-181 family predicts response to concomitant chemoradiotherapy with temozolomide in glioblastoma patients. Neoplasma 57(3), 264-269 (2010).

16 Gonzalez-Gomez P, Sanchez P, Mira H. MicroRNAs as regulators of neural stem cell-related pathways in glioblastoma multiforme. Mol. Neurobiol. 44(3), 235-249 (2011).

17 Zhang W, Zhang J, Hoadley K et al. miR-181d: a predictive glioblastoma biomarker that downregulates $M G M T$ expression. Neuro. Oncol. 14(6), 712-719 (2012).

18 Ujifuku K, Mitsutake N, Takakura S et al. miR-195, miR-455-3p and miR-10a $\left(^{*}\right)$ are implicated in acquired temozolomide resistance in glioblastoma multiforme cells. Cancer Lett. 296(2), 241-248 (2010).

19 Niemoeller OM, Niyazi M, Corradini S et al. MicroRNA expression profiles in human cancer cells after ionizing radiation. Radiat. Oncol. 6, 29 (2011).

20 Sasaki A, Udaka Y, Tsunoda Y et al. Analysis of p53 and miRNA expression after irradiation of glioblastoma cell lines. Anticancer Res. 32(11), 4709-4713 (2012).

21 Reya T, Morrison SJ, Clarke MF, Weissman IL. Stem cells, cancer, and cancer stem cells. Nature 414(6859), 105-111 (2001).

22 Yang YP, Chien Y, Chiou GY et al. Inhibition of cancer stem cell-like properties and reduced chemoradioresistance of glioblastoma using microRNA145 with cationic polyurethane-short branch PEI. Biomaterials 33(5), 1462-1476 (2012).

23 Rutka JT, Weyerbrock A, Liang ML. Gliomas: quo vadis. Clin. Neurosurg. 53, 58-63 (2006). 